\title{
High-Resolution Probe-Based Confocal Laser Endomicroscopy for Diagnosing Biliary Diseases
}

\author{
Hiroki Koda', Kazuo Hara', Okuno Nozomi', Takamichi Kuwahara', Mizuno Nobumasa', Shin Haba', Miyano Akira' and Isomoto \\ Hajime $^{2}$ \\ Department of Gastroenterology, ${ }^{1}$ Aichi Cancer Center Hospital, Nagoya, ${ }^{2}$ Tottori University Hospital, Yonago, Japan
}

Probe-based confocal laser endomicroscopy is an endoscopic technique that enables in vivo histological evaluation using fluorescent pigment. The ability to diagnostically differentiate between benign and malignant biliary disease using the "CholangioFlex ${ }^{\mathrm{TM}}$, a dedicated biliary device, has been reported. However, the Miami and Paris classifications, used as diagnostic criteria, mainly evaluate findings in the submucosa, and visualizing the epithelium as the main site of lesions remains difficult. To address this problem, we verified the imaging findings and diagnostic ability of three types of probes: CholangioFlex ${ }^{\mathrm{TM}}$, GastroFlex ${ }^{\mathrm{TM}}$, and AlveoFlex ${ }^{\mathrm{TM}}$. With GastroFlex $^{\mathrm{TM}}$, the clear mucosal epithelium was observed, and differential diagnoses as benign/malignant could be made based on epithelial findings. GastroFlex ${ }^{\mathrm{TM}}$ may be a good first-choice probe for probe-based confocal laser endomicroscopy of biliary diseases, and a new diagnostic classification based on bile duct epithelial findings may provide useful criteria independent of the Miami or Paris classifications. Clin Endosc 2021;54:924-929

Key Words: Bile duct cancer; Bile duct epithelium; Bile duct stenosis; Confocal laser endomicroscopy; Paris classification

\section{INTRODUCTION}

Although endoscopic diagnosis of biliary disease is performed by endoscopic retrograde cholangiopancreatography (ERCP), endoscopic ultrasonography (EUS), intraductal ultrasonography, and cholangioscopy, the low sensitivity of transpapillary bile duct biopsy and bile juice cytology (BJC), and the high frequency of false-positive results from BJC are well-known clinical problems. Thus, additional endoscopic procedures are often required, and unnecessary surgery may sometimes be needed. ${ }^{1-3}$

Received: July 14, 2020 Revised: November 23, 2020

Accepted: November 23, 2020

Correspondence: Kazuo Hara

Department of Gastroenterology, Aichi Cancer Center Hospital, 1-1 Kanokoden, Chikusa-ku, Nagoya 464-8681, Japan

Tel: +81-52-762-6111, Fax: +81-52-764-2963, E-mail: khara@aichi-cc.jp ORCID: https://orcid.org/0000-0002-4699-6136

(cc) This is an Open Access article distributed under the terms of the Creative Commons Attribution Non-Commercial License (http://creativecommons.org/ licenses/by-nc/3.0) which permits unrestricted non-commercial use, distribution, and reproduction in any medium, provided the original work is properly cited.
Probe-based confocal laser endomicroscopy (pCLE) has recently been anticipated as a new diagnostic tool. CLE is a breakthrough endoscopic technique that uses the fluorescent pigment, fluorescein, to observe cells in vivo, enabling virtual biopsy through real-time histological evaluation. The utility of pCLE for diagnosis of malignant biliary lesions, using the Miami or Paris classifications, has been reported. ${ }^{4-9}$ In previous reports examining the utility of CLE, only the bile duct-specific CholangioFlex ${ }^{\mathrm{TM}}$ (Mauna Kea Technologies, Paris, France) probe was used, and no reports have clarified the diagnostic ability of other types of probes in classifying the lesions. GastroFlex $^{\mathrm{TM}}$ (Mauna Kea Technologies) is supposed to offer high image resolution and is reportedly able to obtain clearer images than other probes. ${ }^{10}$ A classification using detailed imaging findings may lead to further improvements in diagnostic ability.

This study included seven patients who underwent pCLE of bile duct lesions with three types of probes: GastroFlex ${ }^{\mathrm{TM}}$, a dedicated gastrointestinal probe; AlveoFlex ${ }^{\mathrm{TM}}$ (Mauna Kea Technologies), a dedicated lung probe; and CholangioFlex ${ }^{\mathrm{TM}}$. Patients were enrolled from January 2015 to October 2018, 
and pCLE imaging findings and diagnostic ability were evaluated. These cases were part of a retrospective cohort study at a single center, Aichi Cancer Center Hospital in Nagoya, Japan, and was approved by the institutional review board of the hospital (approval number: 2020-1-083).
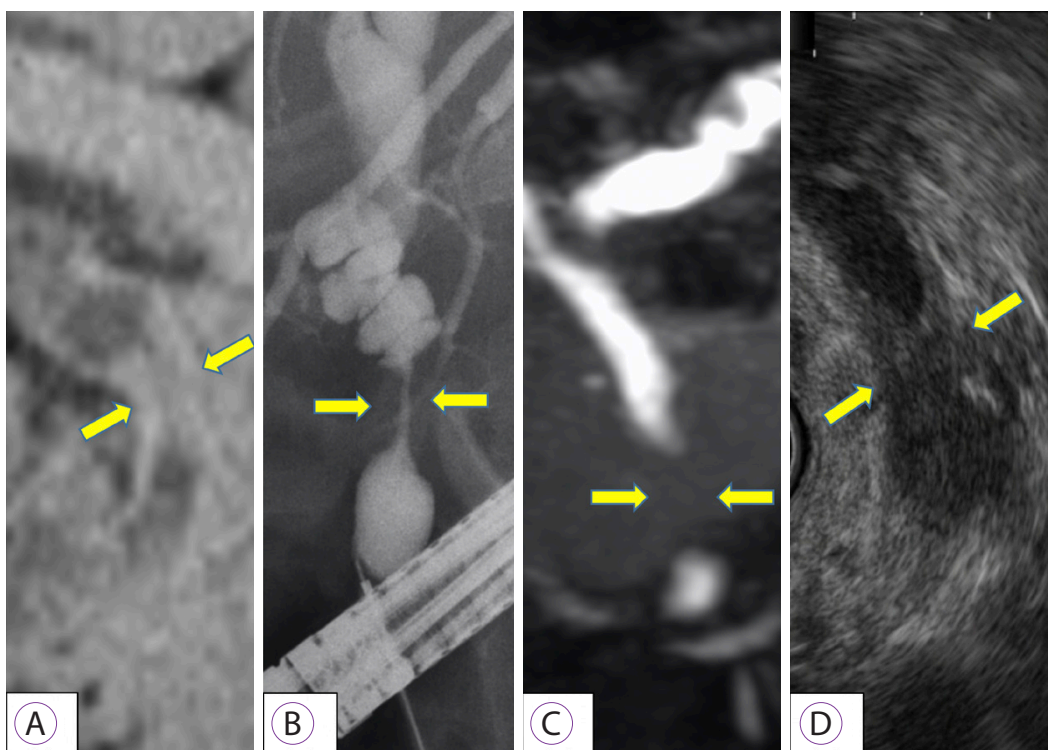

Fig. 1. Imaging findings in bile duct cancer. (A) Computed tomography; (B) endoscopic retrograde cholangiopancreatography; (C) magnetic resonance cholangiopancreatography; (D) endoscopic ultrasonography. Various images show distal bile duct stenosis (yellow arrow) caused by irregular bilateral wall thickness.
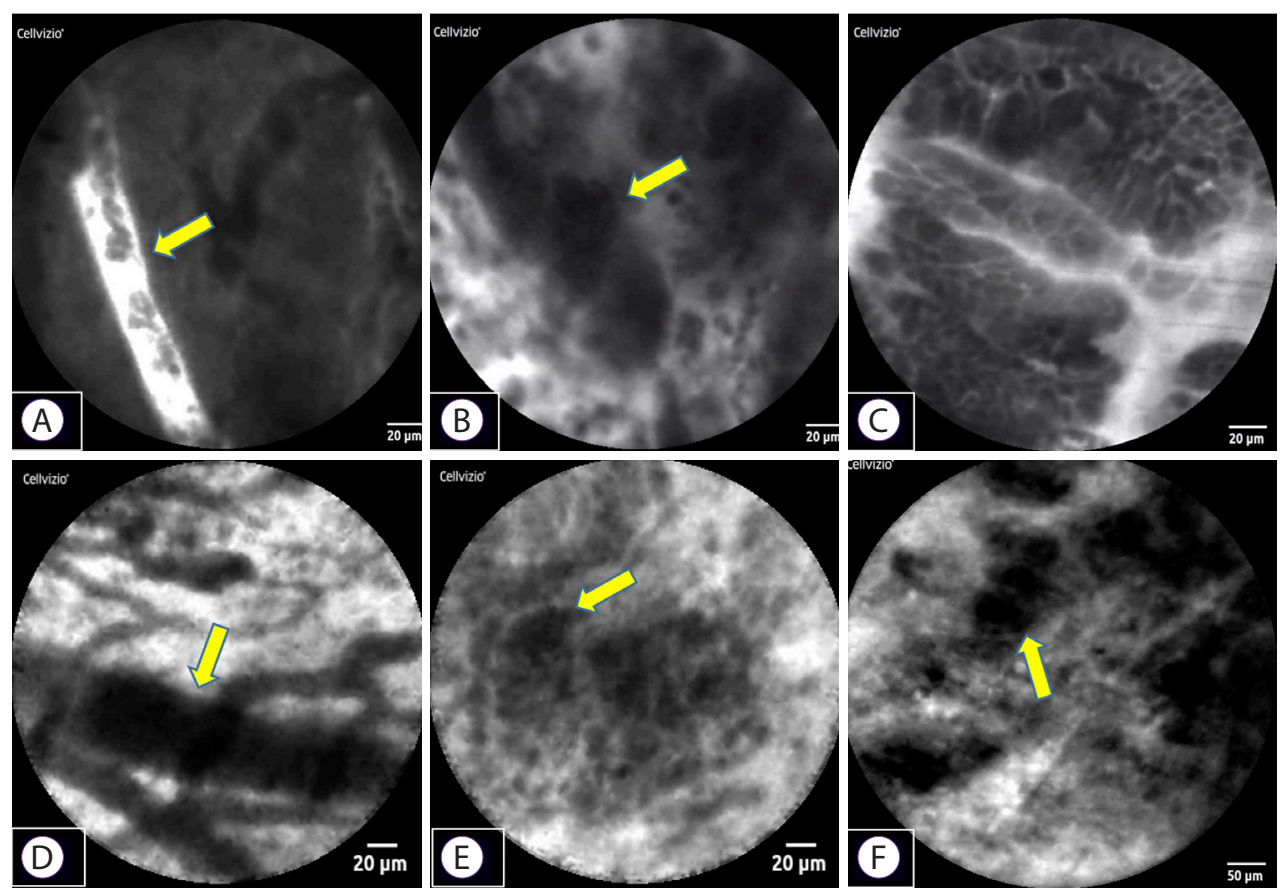

Fig. 2. Representative probe-based confocal laser endomicroscopy (pCLE) images of bile duct cancer. Malignant findings, based on the Miami classification, on pCLE with 3 types of probes produced by Mauna Kea Technologies (Paris, France) ((A-C) GastroFlex ${ }^{\mathrm{TM}},(\mathrm{D}, \mathrm{E})$ CholangioFlex $^{\mathrm{TM}},(\mathrm{F})$ AlveoFlex $^{\mathrm{TM}}$ ) are as follows. (A) Thick white bands; (B) dark clumps; (C) epithelial structure; (D) thick dark bands; (E) dark clumps; (F) dark clumps. 
of the entire circumference of the wall and dilated peripheral bile duct. EUS showed hypoechoic wall thickness at the same site, and the border with pancreatic parenchyma was obscured. ERCP and magnetic resonance cholangiopancreatography showed irregular wall thickness of the entire circumference of the distal bile duct (Fig. 1). We performed pCLE during the ERCP examination. The pCLE probes were inserted into the bile duct through the device channel up to the peripheral site of the lesion, and $2 \mathrm{~mL}$ of fluorescein sodium was injected. Once the fluorescein reached the biliary tissue and the pCLE image was visible, we adjusted the probe position under fluoroscopy. Using the Miami classification (malignant findings: (1) epithelial structures [ES]; (2) thick white bands [TWB]; (3) thick dark bands [TDB]; and (4) dark clumps [DC]), ${ }^{6}$ GastroFlex ${ }^{\mathrm{TM}}$ showed DC, TWB, and ES; CholangioFlex ${ }^{\mathrm{TM}}$ showed TDB and DC; and AlveoFlex ${ }^{\mathrm{TM}}$ showed DC. Most findings were visualized with GastroFlex ${ }^{\mathrm{TM}}$, and malignant findings could be detected with all probes. Moreover, because bile duct epithelial findings were obtained with GastroFlex ${ }^{\mathrm{TM}}$, malignancy could be suspected based on epithelial findings alone, such as cell size difference (CSD), cell overlap (CO), irregular edges of cell clumps (IEC), irregular structures (ISs), and dark epithelium (DE) (Fig. 2). Based on these imaging findings, the preoperative diagnosis was bile duct cancer. Pancreaticoduodenectomy was performed, and the final pathological diagnosis was distal bile duct cancer, as diagnosed preoperatively. Patient background, imaging findings, and pathological findings on endobiliary biopsy/BJC of this case and 6 others are shown in Table 1. In these cases, although the relationship between the time course after injection of fluorescein and the diagnostic ability could not be investigated, we consider that the imaging findings of pCLE with the three probes were not significantly affected by the time course, because the observation time of pCLE was $\leq 50 \mathrm{~min}$ in all cases (corresponding to the so-called $\alpha$ to $\beta$ phases in terms of the elimination half-life of fluorescein). ${ }^{11}$

\section{DISCUSSION}

In this study, the pCLE system "Cellvizio" produced by Mauna Kea Technologies (Paris, France) and three types of probes with different specifications (GastroFlex ${ }^{\mathrm{TM}} /$ CholangioFlex ${ }^{\mathrm{TM}} /$ AlveoFlex ${ }^{\mathrm{TM}}$; compatible operating channel: $\geq 2.8$ $\mathrm{mm} / \geq 1 \mathrm{~mm} / \geq 1.9 \mathrm{~mm}$; field of view: $240 \mu \mathrm{m} / 325 \mu \mathrm{m} / 600$ $\mu \mathrm{m}$; resolution: $1 \mu \mathrm{m} / 3.5 \mu \mathrm{m} / 3.5 \mu \mathrm{m}$; and confocal depth: $55-65 \mu \mathrm{m} / 40-70 \mu \mathrm{m} / 0-50 \mu \mathrm{m}$, respectively) were used; therefore, the characteristics of imaging findings obtained with each probe differed. CholangioFlex ${ }^{\mathrm{TM}}$ showed a collagen fiber network in the submucosa as a normal finding in the bile duct, and image quality was rough and often unclear. In addition, mucosal epithelium could not be confirmed in a routine manner. On the other hand, GastroFlex ${ }^{\mathrm{TM}}$ offered 3.5-times better image resolution than CholangioFlex ${ }^{\mathrm{TM}}$; therefore, more detailed images could be visualized, as previously reported..$^{10}$ In particular, the characteristics of the mucosal epithelium were clearly evident, and the greatest merit of GastroFlex ${ }^{\mathrm{TM}}$ was its ability to evaluate information at both the mucosal epithelium and the submucosal layer. However, bile duct cannulation with GastroFlex $^{\mathrm{TM}}$ is slightly more difficult because the outer diameter of the probe is more than twice that of CholangioFlex ${ }^{\mathrm{TM}}$. As a result, this device requires an additional learning curve. AlveoFlex ${ }^{\mathrm{TM}}$ offers a wide visual field of $600 \mu \mathrm{m}$, making the judgment of small structures difficult; therefore, identifying malignant findings, including epithelial involvement, was more difficult with this probe as compared to the other two. In terms of diagnostic ability, based on the Miami classification, although the number of cases was small (seven cases), the sensitivity, specificity and accuracy of CholangioFlex ${ }^{\mathrm{TM}}$, GastroFlex ${ }^{\mathrm{TM}}$, and AlveoFlex ${ }^{\mathrm{TM}}$ were $75 \%, 100 \%$, and $75 \% ; 66.7 \%$, $33.3 \%$, and $33.3 \%$; and $71.4 \%, 71.4 \%$, and $57.1 \%$, respectively. Similar to existing reports, ${ }^{9}$ the specificity was low, and no significant differences were observed in the diagnostic ability of the three probes (Table 1). Based on the above results, it remains unclear whether the Miami classification (as a set of diagnostic criteria using CholangioFlex ${ }^{\mathrm{TM}}$ ) can be applied to the other probes used in this study.

Here, we focused on epithelial findings that could be observed with GastroFlex ${ }^{\mathrm{TM}}$, which enabled us to obtain detailed images. With the other two probes, epithelial findings were observed in only a single case (14.3\%) each, whereas with GastroFlex ${ }^{\mathrm{TM}}$, epithelial findings were observed in all cases due to the high image resolution. Since these results showed that using GastroFlex ${ }^{\mathrm{TM}}$ significantly $(p=0.005)$ improved the ability to observe epithelial findings (Table 1), we consider that using epithelial findings to differentiate between benign and malignant lesions could lead to further improvement of the ability of pCLE to diagnose biliary diseases. On the other hand, lesions deeper than the submucosa, such as extraepithelial tumor extensions, cannot be diagnosed by pCLE. Therefore, other examinations, such as EUS-guided fine needle aspiration, may be necessary in some cases.

We established new diagnostic criteria for epithelial findings from pCLE and evaluated its diagnostic ability. The following five findings were suggestive of malignancy: (1) CSD, where each cell is heterogeneous and differs in size; (2) CO, where epithelial cells that are normally present in monolayers appear to overlap instead; (3) IEC, where cell clumps are irregular owing to the ubiquity of the nucleus and weakening of cell adhesion molecules; (4) IS, where epithelial cell composition 


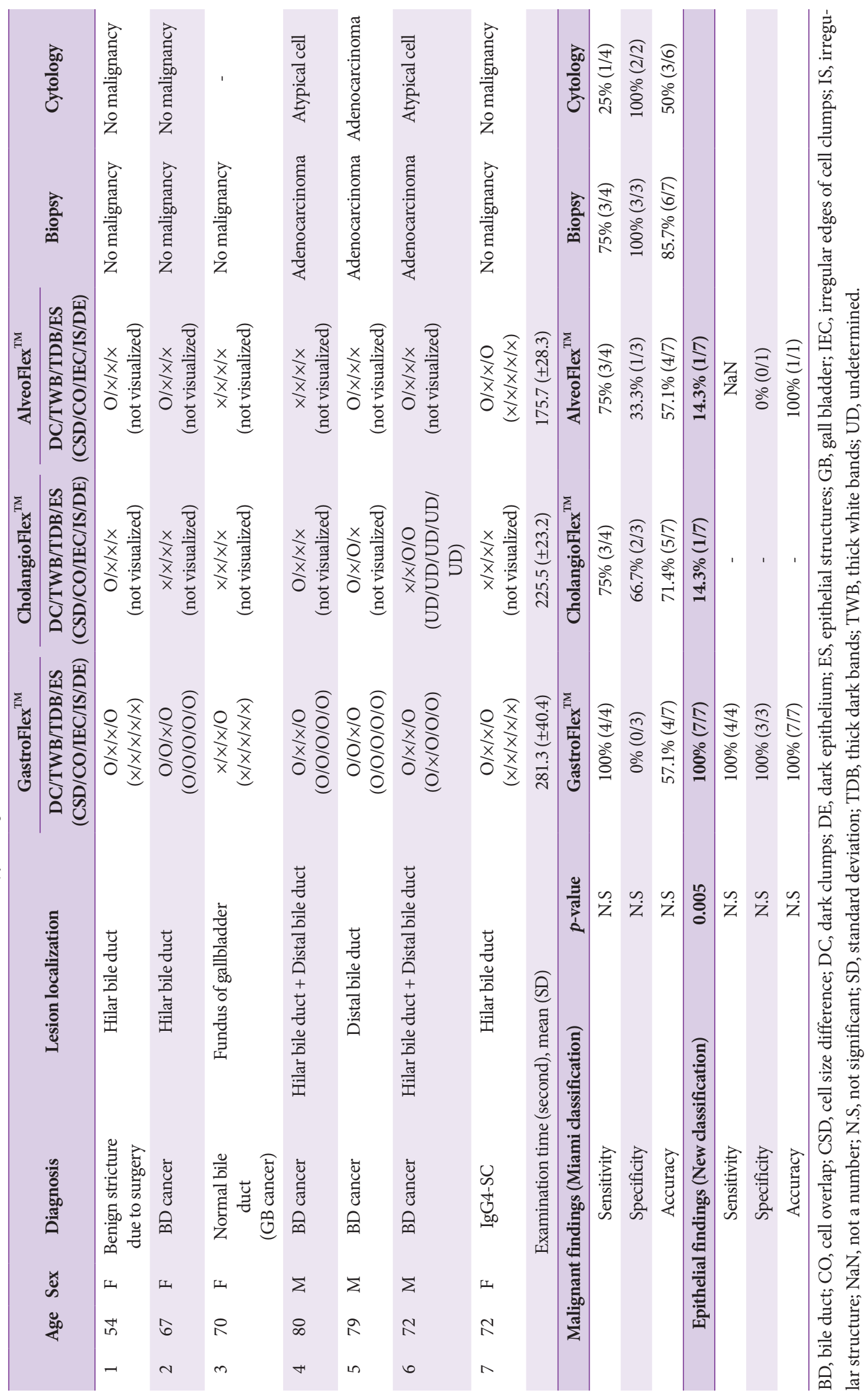



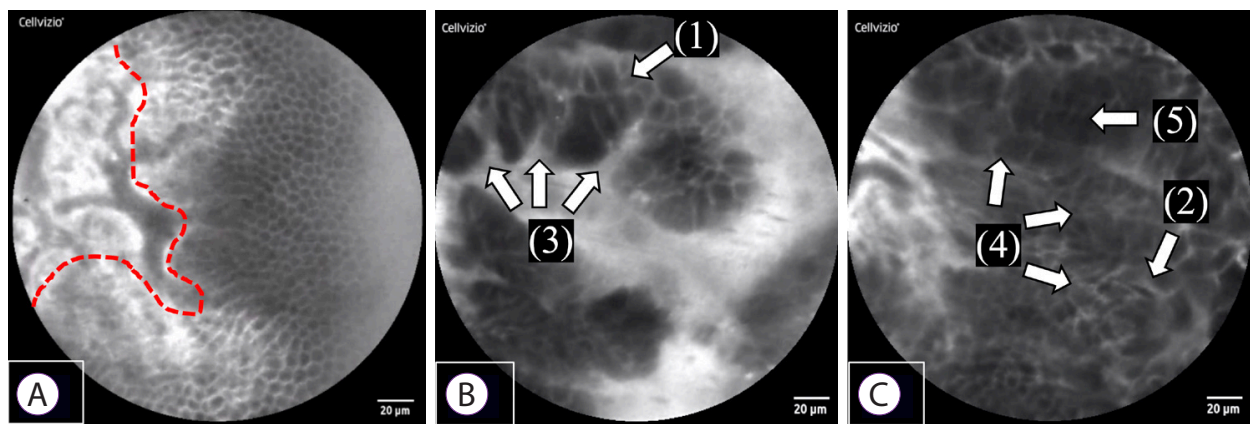

Fig. 3. Normal and malignant findings in epithelial structures. (A) Normal bile duct: a sheet-like monolayer of uniform epithelial cells is arrayed on the right side, and a collagen fiber network in the submucosa is presented on the left side (the red dotted line represents the border). (B, C) Bile duct cancer: (1) Each cell is heterogeneous, and the cells differ in size: cell size difference, (2) Epithelial cells appear to overlap: cell overlap, (3) Irregularity of cell clumps: irregular edges of cell clumps, (4) Irregular and complex epithelial cell composition: irregular structure, (5) The nucleus and cytoplasm cannot be distinguished as the entire cell appears dark: dark epithelium.
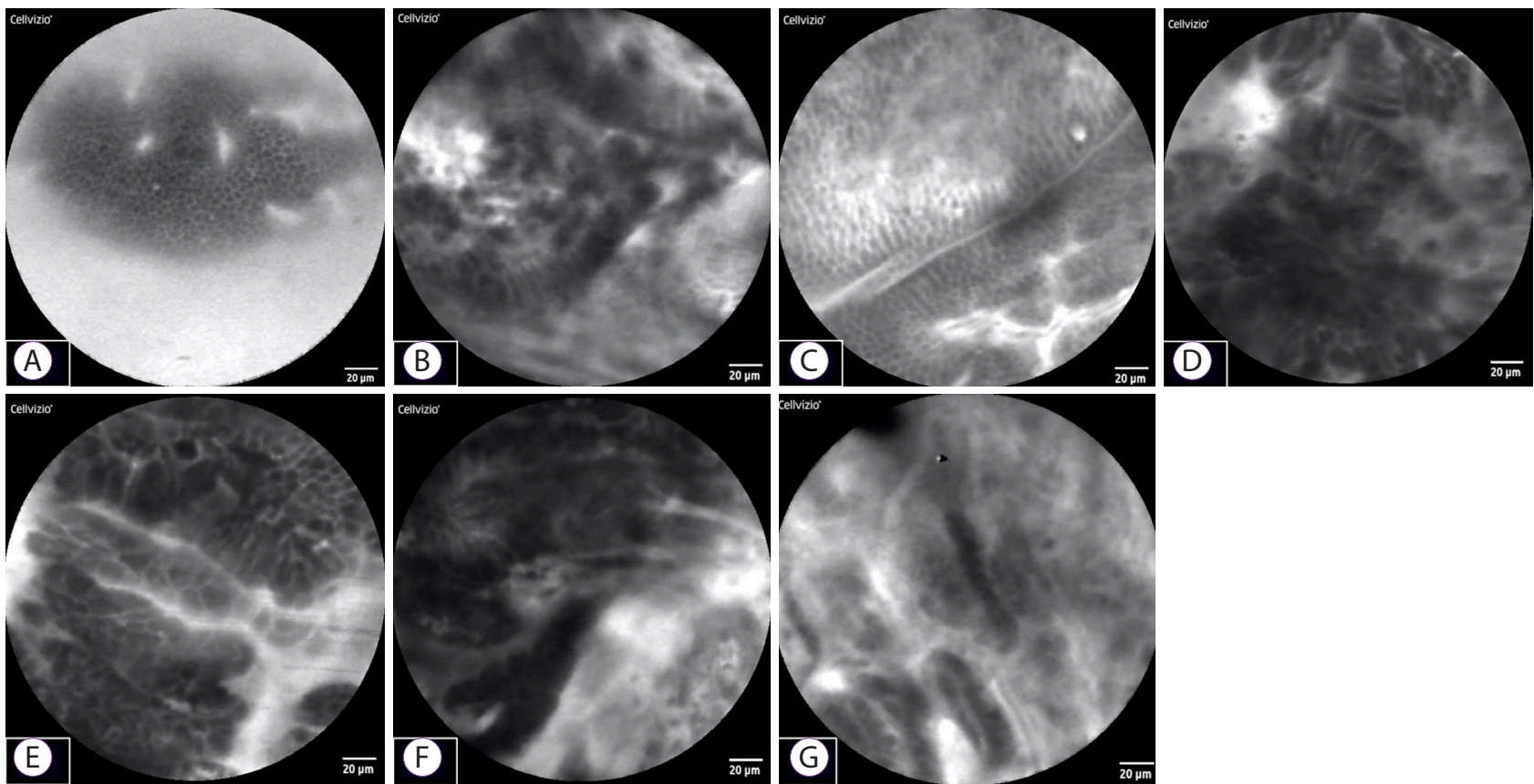

Fig. 4. Representative probe-based confocal laser endomicroscopy epithelial findings in 7 cases. (A) Cases 1 and 3: benign stricture due to surgery. Sheet-like monolayer of uniform epithelial cells. (B, D, E, F) Cases 2, 4, 5, and 6: bile duct cancer. Each cell is heterogeneous, and cells overlap and differ in size. (C) Case 3: normal bile duct. Sheet-like monolayer of uniform epithelial cells. (G) Case 7: IgG4-related sclerosing cholangitis. Cells are not sheet-like, but are relatively uniform in size, with no apparent overlap.

is irregular and complex; and (5) DE, where the nucleus and cytoplasm cannot be distinguished, as the entire cell appears dark (Fig. 3). On examining the pCLE findings in the seven patients, all of the above five new diagnostic criteria were observed in three out of the four cases of bile duct cancer, while the fourth case had malignant findings of both CSD and IEC (Fig. 4). The sensitivity was $100 \%$, indicating the utility of this method for diagnosing malignancy. In addition, malignant epithelial findings were not observed in the three cases of benign disease that showed false-positive results according to the Miami classification; therefore, specificity was also 100\% (Table $1)$.

As described above, pCLE using epithelial findings shows excellent diagnostic ability, and since epithelial findings themselves can also be easily compared with pathological findings, GastroFlex ${ }^{\mathrm{TM}}$ should be the first choice for pCLE of biliary 
diseases. However, pCLE does not provide findings about the cell nucleus, which is a very important factor in the diagnosis of malignancy; hence, we look forward to the development of fluorescent pigments that can enable the identification of nuclear findings.

In this study, three types of probes were used to diagnose biliary lesions. Since only a small number of studies have been reported, the most useful probe remains unclear, but GastroFlex ${ }^{\mathrm{TM}}$ impressively provided markedly clearer images than other probes. We consider the clarity of images from GastroF$\mathrm{lex}^{\mathrm{TM}}$ as essential to achieve high diagnostic ability. Diagnosis with pCLE based solely on epithelial findings using GastroF$\mathrm{lex}^{\mathrm{TM}}$, without relying on the existing Miami classification of unclear submucosal findings, is expected to provide a useful new method for diagnosing biliary diseases. Accumulation of pCLE cases examined using GastroFlex ${ }^{\mathrm{TM}}$ is desirable, and diagnostic ability (including which of the epithelial findings are very important) must be verified in a larger number of cases.

\section{Conflicts of Interest}

Mizuno Nobumasa reports grants and personal fees from Novartis. The authors have no potential conflicts of interest.

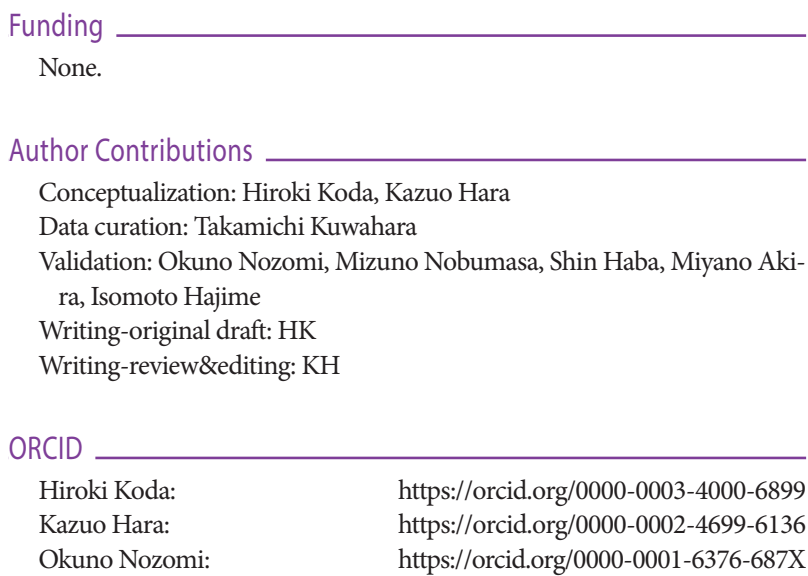

Takamichi Kuwahara: Mizuno Nobumasa: Shin Haba: Miyano Akira: Isomoto Hajime: https://orcid.org/0000-0001-8348-8926 https://orcid.org/0000-0001-9704-1885 https://orcid.org/0000-0002-7578-5610 https://orcid.org/0000-0001-7313-0349 https://orcid.org/0000-0001-8998-7865

\section{REFERENCES}

1. Ponchon T, Gagnon P, Berger F, et al. Value of endobiliary brush cytology and biopsies for the diagnosis of malignant bile duct stenosis: results of a prospective study. Gastrointest Endosc 1995;42:565-572.

2. Schoefl R, Haefner M, Wrba F, et al. Forceps biopsy and brush cytology during endoscopic retrograde cholangiopancreatography for the diagnosis of biliary stenoses. Scand J Gastroenterol 1997;32:363-368.

3. Rösch T, Hofrichter K, Frimberger E, et al. ERCP or EUS for tissue diagnosis of biliary strictures? A prospective comparative study. Gastrointest Endosc 2004;60:390-396.

4. Meining A, Chen YK, Pleskow D, et al. Direct visualization of indeterminate pancreaticobiliary strictures with probe-based confocal laser endomicroscopy: a multicenter experience. Gastrointest Endosc 2011;74:961-968.

5. Caillol F, Filoche B, Gaidhane M, Kahaleh M. Refined probe-based confocal laser endomicroscopy classification for biliary strictures: the Paris classification. Dig Dis Sci 2013;58:1784-1789.

6. Wallace M, Lauwers GY, Chen Y, et al. Miami classification for probebased confocal laser endomicroscopy. Endoscopy 2011;43:882-891.

7. Kahaleh M, Giovannini M, Jamidar P, et al. Probe-based confocal laser endomicroscopy for indeterminate biliary strictures: refinement of the image interpretation classification. Gastroenterol Res Pract 2015;2015:675210.

8. Slivka A, Gan I, Jamidar P, et al. Validation of the diagnostic accuracy of probe-based confocal laser endomicroscopy for the characterization of indeterminate biliary strictures: results of a prospective multicenter international study. Gastrointest Endosc 2015;81:282-290.

9. Meining A, Shah RJ, Slivka A, et al. Classification of probe-based confocal laser endomicroscopy findings in pancreaticobiliary strictures. Endoscopy 2012;44:251-257.

10. Shieh FK, Drumm H, Nathanson MH, Jamidar PA. High-definition confocal endomicroscopy of the common bile duct. J Clin Gastroenterol 2012;46:401-406

11. Blair NP, Evans MA, Lesar TS, Zeimer RC. Fluorescein and fluorescein glucuronide pharmacokinetics after intravenous injection. Invest Ophthalmol Vis Sci 1986;27:1107-1114 